\title{
Análisis de las estrategias bioclimáticas empleadas por Frank Lloyd Wright en la casa Jacobs I
}

\author{
Analysis of the bioclimatic strategies used by Frank Lloyd Wright \\ in the Jacobs I house
}

$\underline{\text { M. Beltrán-Fernández }}{ }^{(*)}$, J. García-Muñoz $^{(*)}$, E. Dufrasnes ${ }^{(*)}$

\section{RESUMEN}

Frank Lloyd Wright es considerado uno de los arquitectos más influyentes de la historia de la arquitectura moderna por la calidad espacial y estética de su obra. Sin embargo, la importancia de sus soluciones constructivas y medioambientales ha quedado en un segundo plano. Con el fin de destacar dichas cualidades se ha analizado la primera casa que diseñó para la familia Jacobs (Wisconsin, EE.UU., 1937), cuyo valor arquitectónico ha sido resaltado en varias ocasiones, sin que existan análisis que cuantifiquen las ventajas sobre la calidad medioambiental y el confort interior que suponen los principios bioclimáticos de su arquitectura. Pero ¿cómo medir la influencia de la arquitectura bioclimática en el confort interior? ¿Supone una mejora la orientación elegida? ¿Y la influencia de la ventilación natural y de los voladizos? Para responder a estas cuestiones se ha construido un modelo digital y se han realizado diferentes simulaciones energéticas empleando Revit y Design Builder.

Palabras clave: arquitectura bioclimática; Frank Lloyd Wright; casa Jacobs I; calidad medioambiental; confort térmico; simulación energética.

\section{ABSTRACT}

Frank Lloyd Wright is considered one of the most influential architects of modern architecture due to the spatial and aesthetic qualities of his work. However, the importance of Wright's construction and environmental solutions has been usually overlooked. In order to evaluate these qualities, the first house that Wright designed for the Jacobs family (Wisconsin, USA, 1937) has been analyzed. This well-known building has been much studied, and its architectural value much celebrated, but without analysing the advantages of the bioclimatic principles over interior environment. But how can the influence of this bioclimatic architecture be measured in terms of thermal comfort? Does the selected orientation improve interior comfort? And what about the natural ventilation or the deep overhangs? In order to assess this, a digital model for energy simulation has been built using Revit and Design Builder.

Keywords: bioclimatic architecture; Frank Lloyd Wright; house Jacobs I; environmental quality; thermal comfort; energy simulation.

\footnotetext{
(*) Escuela Técnica Superior de Edificación - Universidad Politécnica de Madrid. Madrid (España).

${ }^{(* *)}$ Ecole Nationale Supérieure d'Architecture - Université Strasbourg. Estrasburgo (France).

Persona de contacto/Corresponding author: mariangeles.beltran@upm.es (M. Beltrán-Fernández)

ORCID: http://orcid.org/oooo-0oo2-4680-9732 (M. Beltrán-Fernández); http://orcid.org/oooo-0002-9924-7593

(J. García-Muñoz); http://orcid.org/oooo-0002-7341-8470 (E. Dufrasnes)
}

Cómo citar este artículo/Citation: Beltrán-Fernández, M., García-Muñoz, J., Dufrasnes, E. (2017). Análisis de las estrategias bioclimáticas empleadas por Frank Lloyd Wright en la casa Jacobs I. Informes de la Construcción, 69(547): e213, doi: http://dx.doi. org/10.3989/ic.16.156.

Copyright: (C) 2017 CSIC. Licencia / License: Salvo indicación contraria, todos los contenidos de la edición electrónica de Informes de la Construcción se distribuyen bajo una licencia de uso y distribución Creative Commons Attribution License (CC BY) Spain 3.o. 


\section{INTRODUCCIÓN}

Frank Lloyd Wright (1867-1959) es considerado uno de los arquitectos más influyentes de la historia de la arquitectura moderna por la calidad espacial y estética de su obra como demuestran la famosa casa de la Cascada (Pensilvania, EE.UU., 1935-1939), o el museo Guggenheim (Nueva York, EE.UU., 1943-1959). Sin embargo, en numerosas ocasiones la importancia e innovación de sus soluciones constructivas y medioambientales han quedado en un segundo plano y merece ser analizada (1) (2).

Wright consagró su carrera a la búsqueda constante de una tipología de vivienda confortable y accesible económicamente para la familia media americana. Esta vivienda de tamaño moderado, que el arquitecto denominó Usonian House (3), debía despojarse de todo aquello que no fuera imprescindible, y ser construida con materiales sencillos y en su estado natural, respondiendo a los condicionantes climatológicos del lugar para lograr un confort interior adecuado, dentro de una lógica constructiva que en la actualidad se conoce como sostenibilidad (4). La casa Jacobs I representa el primer prototipo de casas usonianas, de las que Wright diseño más de 150 unidades a lo largo de su carrera. En todas ellas aplicó de forma sistemática las estrategias bioclimáticas analizadas a continuación, apoyándose en el desarrollo de materiales y sistemas constructivos que supuso una mejora en el confort interior de las viviendas.

\section{DESCRIPCIÓN DEL OBJETO DE ESTUDIO. ANÁLISIS CONSTRUCTIVO Y BIOCLIMÁTICO}

\subsection{Situación, clima y programa}

La familia Jacobs conoce a Frank Lloyd Wright en 1936, fecha en la que se mudan a Madison y encargan a Wright una vivienda modesta, de bajo presupuesto, acorde a su modo de vida. A Wright, reputado arquitecto por proyectos como la casa de la Cascada, le entusiasmó la idea, que le permitió experimentar con su modelo de casa usoniana (5).

Madison se encuentra en Wisconsin, al norte de Estados Unidos ( $\left.43^{\circ} 3^{\prime} 31^{\prime \prime} \mathrm{N}, 89^{\circ} 26^{\prime} 29^{\prime \prime} \mathrm{W}\right)$. Posee un clima continental húmedo, con temperaturas máximas en verano que oscilan entre 26 y $32^{\circ} \mathrm{C}$ y en invierno descienden hasta $-23^{\circ} \mathrm{C}$. Las precipitaciones anuales son de $800 \mathrm{~mm}$, siendo más abundantes en los meses estivales. Los vientos dominantes provienen del SW a NE en invierno y del SE a NW en verano (6), como muestran las Figuras 1a y 1b.
El programa se organiza en una planta con un pequeño sótano para las instalaciones de calefacción y agua caliente sanitaria. La planta baja, con una superficie de 1.550 pies cuadrados $\left(144 \mathrm{~m}^{2}\right)$, se dispone en forma de L, albergando los diferentes usos distribuidos en dos alas. El ala paralela a la vía pública alberga la zona vividera orientada al este con vistas al jardín interior a través de las grandes superficies acristaladas del salón. La otra ala contiene los tres dormitorios, con grandes ventanales orientados al jardín sur y conectados entre sí a través del pasillo norte. La charnela entre las dos alas de la vivienda está integrada por el núcleo de cocina y baño, e instalaciones en el sótano, próximos entre sí para su aprovechamiento (Figuras 2a y 2b).

\subsection{Análisis constructivo (7)}

La vivienda, construida sobre una solera de hormigón, se ubica en la esquina noroeste de la parcela, coincidiendo con la parte más elevada. Sobre la solera se representaron los ejes de una malla constructiva de 2'-0" × 4'-0" $(60,9 \times 122 \mathrm{~cm})$, que Wright denominó unity system, al cual obedecían todos los elementos constructivos de la vivienda (5).

Los materiales utilizados son ladrillo y madera. La fábrica de ladrillo para el núcleo de la vivienda conforma la chimenea, cocina, baño y el muro sur del salón. Los ladrillos provenían de los descartes de la obra del edificio administrativo de la $\mathrm{S}$. C. Johnson, también en construcción, para disminuir los costes (8). El cerramiento exterior (Figuras 3 y 4) está compuesto por un nuevo sistema diseñado por el arquitecto a base de tableros y listones de madera con un espesor total de 3 " (7,6 $\mathrm{cm})$, resultando bastante esbelto.

La cubierta de la vivienda presenta tres niveles diferentes; el ala de dormitorios y garaje abierto con una altura de 7'-o" (213 cm), el salón comedor de 9'-o" (274 cm) y el núcleo central con de 11'-o" (335 cm). La estructura está compuesta por tres vigas de madera de $2 \times 4$ " superpuestas, con un canto total de 12" (27 cm canto final). Respetando el módulo de 2'o" $(60,9 \mathrm{~cm})$, apoyan directamente sobre el muro de fábrica o el cerramiento de madera formando los grandes voladizos tan característicos de Wright, de hasta 4'-o" (122 cm), que disminuyen su espesor a medida que aumenta el vuelo en relación a la fachada.

La diferencia de altura entre los niveles de cubiertas se aprovecha para colocar ventanas que permiten iluminar y ventilar los espacios interiores de la casa (Figura 2b).
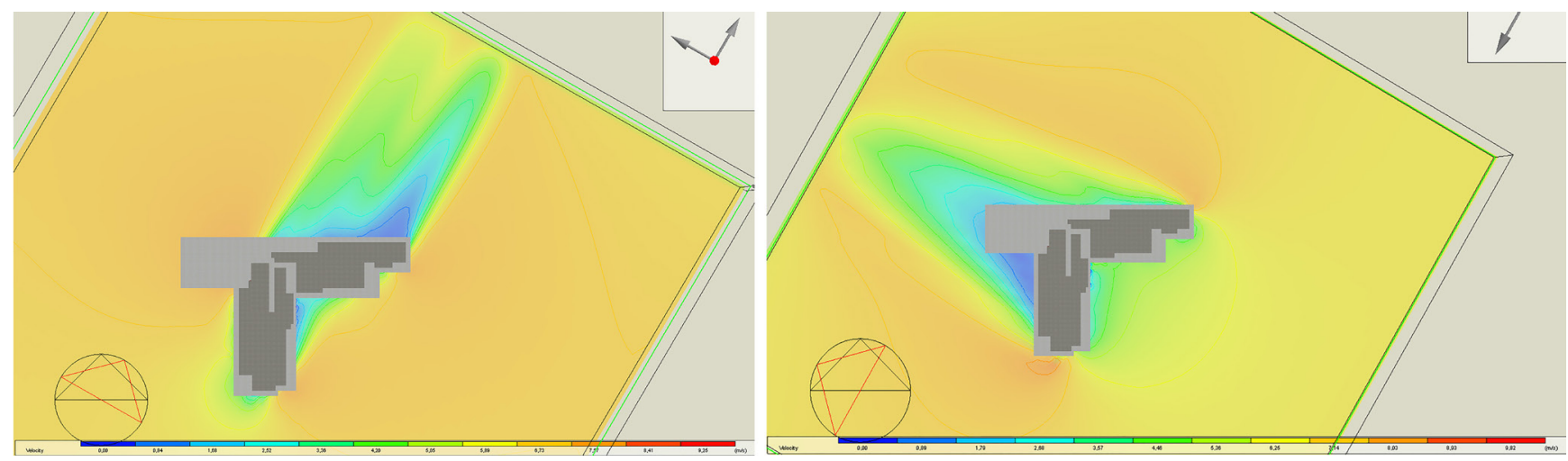

Figuras 1a y 1b. Dirección de los vientos dominantes invierno y verano. 

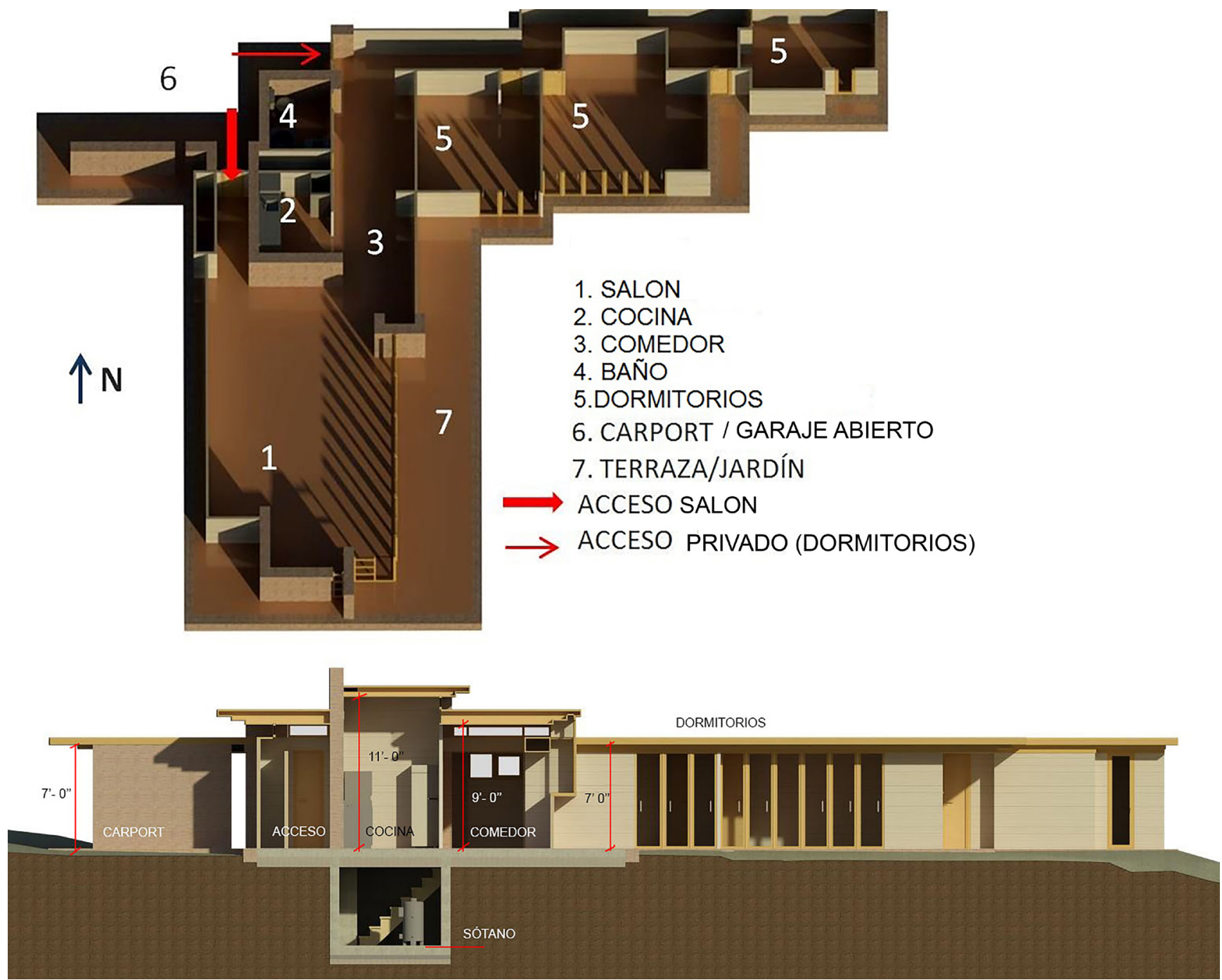

Figuras 2a y 2b. Planta baja y sección.

La carpintería exterior de madera respeta el módulo existente, estructurándose cada 2'-o" con unos pilares de $2 \times 4$ ", coincidiendo con las vigas de cubierta. La carpintería de las fachadas este y sur comprende toda la altura de la vivienda, 7'-o" para los dormitorios y 9'-o" para las puertas del salón, todas practicables, abriéndose completamente a la naturaleza. Sin embargo, las ventanas de las fachadas norte y oeste, con una altura de 1'-o" (30,5 cm) y dispuestas en horizontal con divisiones cada 2'-o", están directamente en contacto con la cubierta. Los vidrios originales de las ventanas provenían de la reutilización de escaparates de locales comerciales, cuidadosamente cortados y limpiados (8), lo cual supuso un ahorro en el coste de ejecución (9).

El sistema de calefacción, al que Wright denominó «Gravity Heat» (Figura 3a), fue el primero por suelo radiante construido en Estados Unidos (5) (10). Se instalaron una serie de circuitos cerrados embebidos en la capa de grava, bajo la solera de hormigón; tres para el ala de dormitorios y cuatro para el salón, compuestos por tuberías de hierro soldadas, de 2" (5 cm) de diámetro, que transportaban vapor de agua calentando la solera y el interior de la vivienda. La fuente de
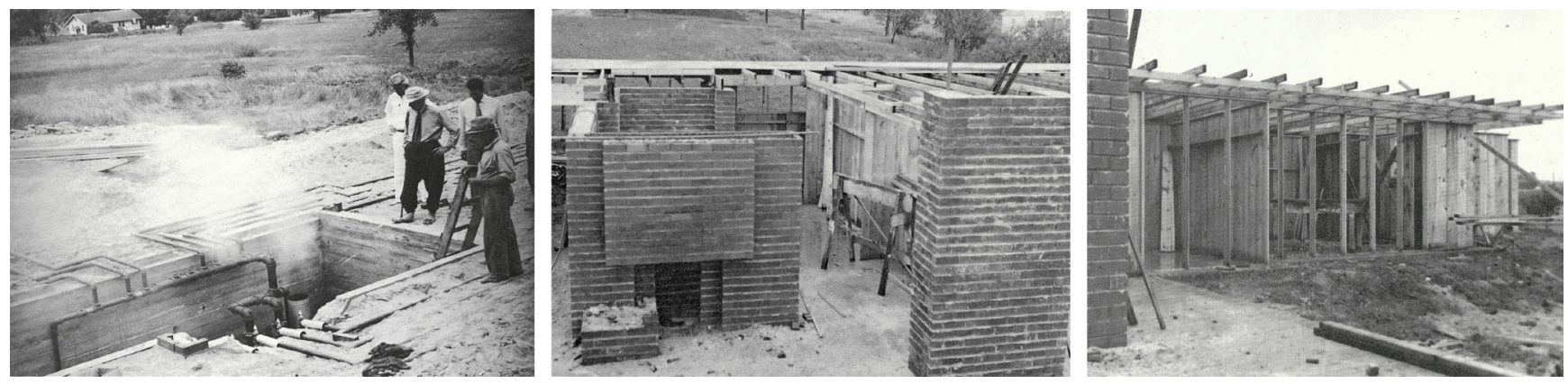

Figuras 3a, 3b y 3c. Construcción de la vivienda: sistema de calefacción «Gravity Heat», chimenea-núcleo central y estructura de madera del ala de dormitorios (Imágenes empleadas con la autorización de la familia Jacobs) (5). 

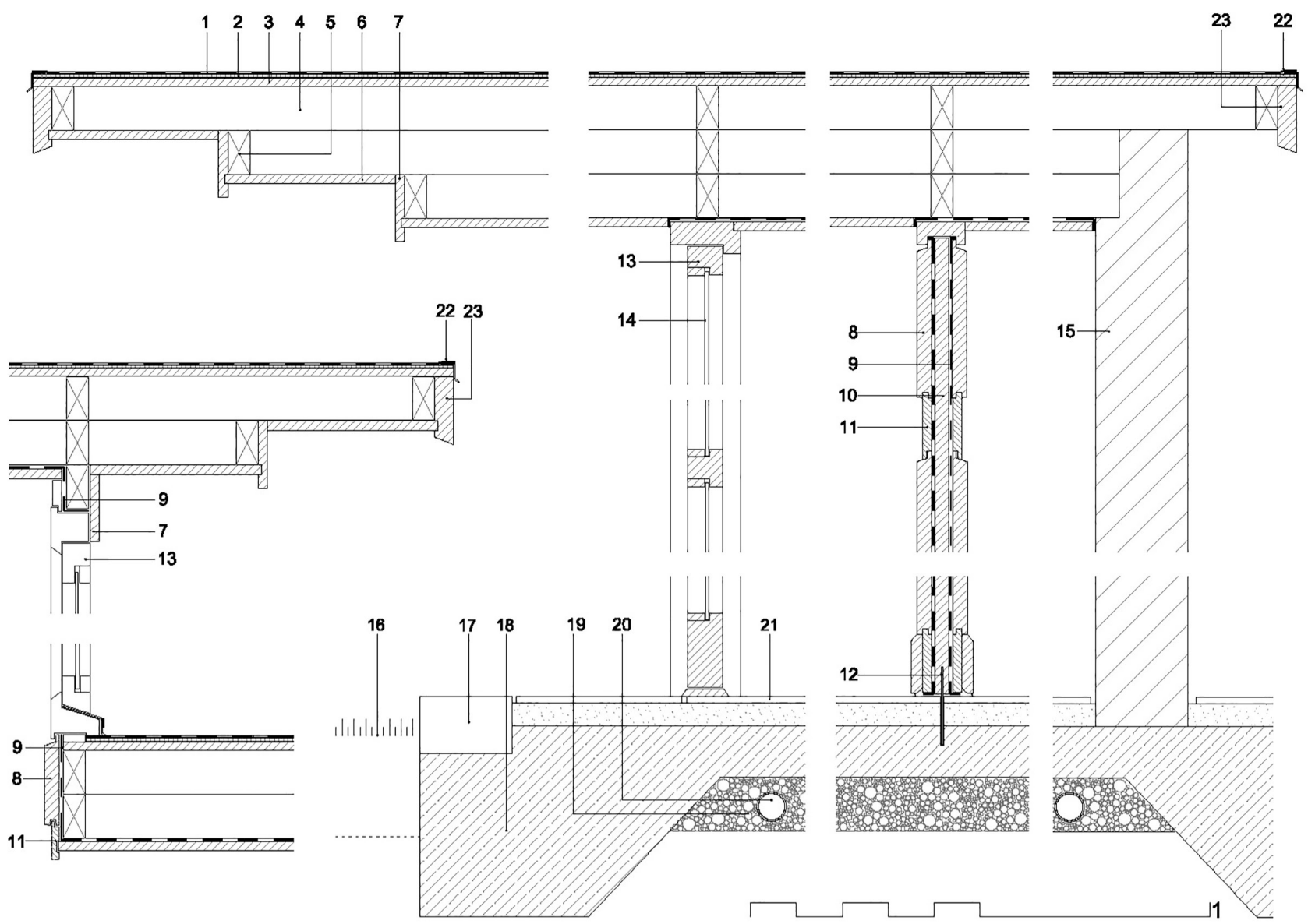

\begin{abstract}
1._Lámina asfáltica; 2._Imprimación; 3._Tablero de pino de $1^{\prime \prime}$ de espesor; 4._Tres vigas de pino superpuestas de 2"x 4"; 5._Zuncho de madera de $2^{\prime \prime} \times 4$ "; 6. Falso techo de tablero de madera de pino de 1" de espesor; 7. Tabica de madera de 1" $\times 31 / 2$ de sección; 8 . Liston de madera de pino de $1^{\prime \prime}$ de espesor con protección exterior aceite de linaza, protección interiór cera; 9._Papel Kraft con imprimación asfáltica; 10._Tablero central de madera de pino de $1^{\prime \prime}$ de espesor; 11._Listones de madera de secuoya atornillados al tablero central; 12 ._Llave de acero galvanizado de soporte $y$ anclaje de cerramiento de madera; 13. Carpintería de madera de pino; 14. Acristalamiento sencillo 4 mm; 15 . Fabrica de ladrillo macizo de 1'-0" de espesor; 16._Terreno vegetal; 17._Hilera de ladrillo a sardinel como encofrado de solera; 18._Solera de hormigón de 4" de espesor; 19._Encachado de grava de $4^{\prime \prime}$ de espesor; 20._Tuberla Gravity Heat $\emptyset 2 "$; 21._ Acabado de resina; 22._Remate de acero galvanizado; 23._Imposta de madera de $2 y_{2}^{\prime \prime} \times 7 y_{2}^{\prime \prime}$ de sección.
\end{abstract}

Figura 4. Detalles constructivos.

generación de calor del sistema consistía en una caldera de gasóleo situada en el sótano (5) (11) (12).

\subsection{Análisis de confort y ambiental}

El diseño y la orientación de la vivienda están fuertemente condicionados por las características climáticas del estado de Wisconsin, como demuestra el tratamiento diferente que Wright otorga a las fachadas (Figuras 5 a y $5 \mathrm{~b}$ ):

- Fachadas sur-este acristaladas y abiertas al jardín.

- Fachadas norte-oeste prácticamente ciegas.

Además del soleamiento, Wright tuvo en cuenta la dirección de los vientos dominantes para la elección de la orientación y la disposición en L de la vivienda, lo cual representaba grandes ventajas.

Como se aprecia en el esquema de soleamiento del solsticio de invierno (Figura 6a), tanto el ala de la zona vividera como los dormitorios, con orientaciones este y sur, se abren al jardín a través de la fachada acristalada aprovechando las ventajas del sol en cuanto a calefacción e iluminación naturales. Wright tuvo en cuenta la inclinación del sol en invierno para la dimensión y retranqueo de los voladizos, permitiendo, en invierno, la entrada de radiación solar en el interior de las estancias (Figura 6a). La zona de los dormitorios queda protegida de la fachada fría norte mediante el pasillo que los comunica. El sistema de calefacción compuesto por el sol, el sistema «Gravity Heat», la chimenea y el aprovechamiento de la inercia térmica de la solera de hormigón y la fábrica de ladrillo, resultaba uno de los sistemas de calefacción más agradables, según la propietaria, Katherine Jacobs (5), afirmando que el confort interior en días soleados podía alcanzarse con $10^{\circ} \mathrm{F}$ menos $\left(5,5^{\circ} \mathrm{C}\right)$ que en una vivienda convencional. Sin embargo, en los días gélidos de invierno, un jersey y la chimenea eran imprescindibles. En cuanto al jardín Wright planificó una serie de árboles para proteger la vivienda de los fríos vientos invernales, predominantes del SW.

En verano las fachadas este y sur reciben radiación solar durante gran parte del día (Figura 6b). Sin embargo, a medida que el sol se eleva las superficies acristaladas quedan protegidas por los grandes voladizos (Figura 6b). Wright orientó la vivienda en función de los vientos dominantes: en verano, para evitar la acumulación de aire caliente en el interior, todas las ventanas son practicables y a través de la ventilación natural cruzada y nocturna consigue una disminución de temperaturas tanto en 

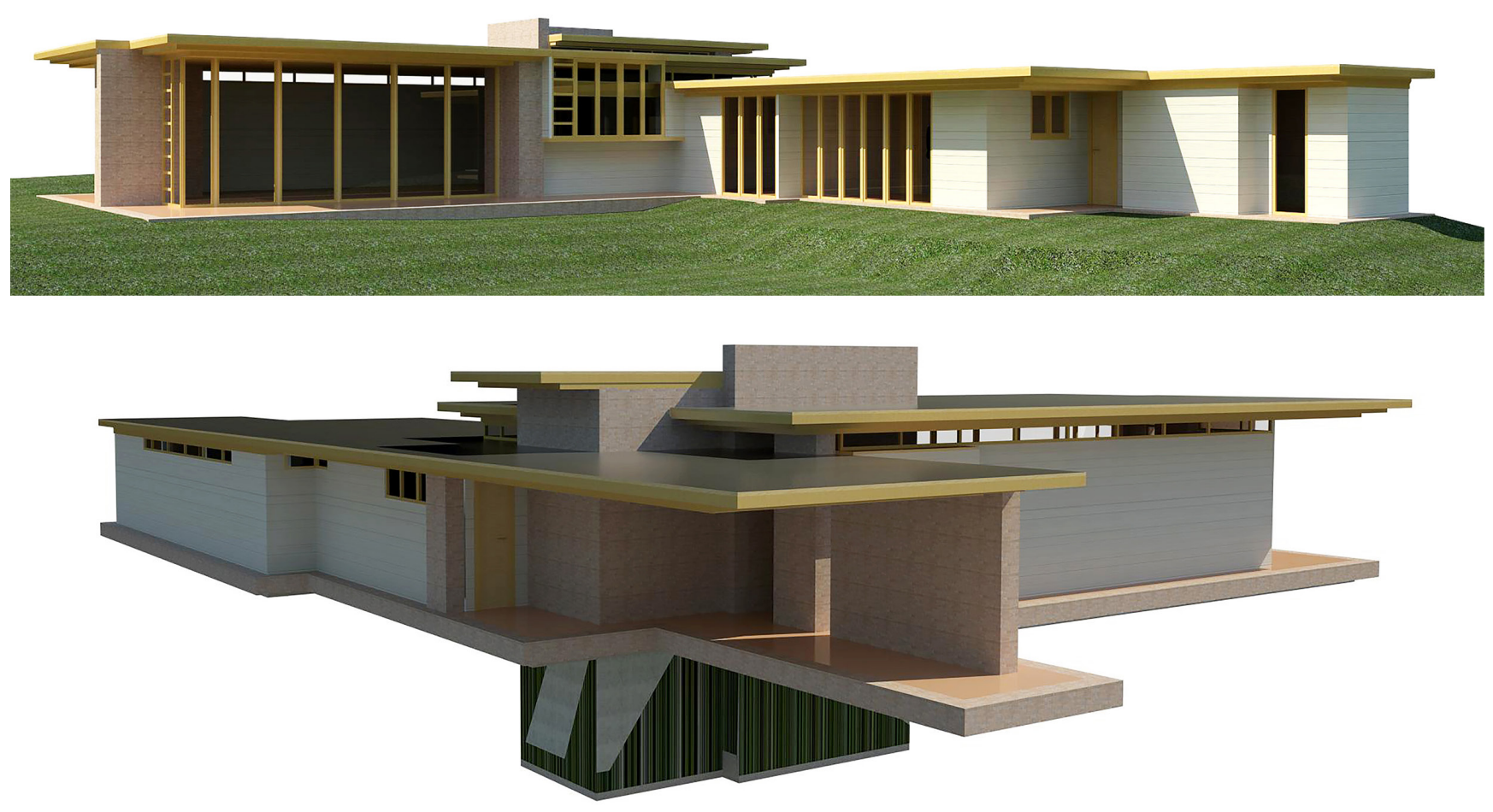

Figuras 5a y 5b. Fachadas sur-este y norte-oeste (21 marzo 14:0o h).

el salón como en los dormitorios. Igualmente se sirvió de la inercia térmica de los materiales; la solera de hormigón y la masa de ladrillo del núcleo central se enfrían durante la noche ayudados por la ventilación natural, refrescando el interior de la vivienda durante las primeras horas del día.

La disposición de la cocina abierta al espacio del comedor y conectada visualmente al jardín a través de la ventana corrida suponía una novedad frente a la tipología residencial de cocina cerrada de la época. Aprovechando la diferencia de niveles de las cubiertas, Wright diseña una serie de ventanas altas orientadas al sur y este que aportan iluminación natural y permiten ventilar la cocina y el baño, evitando que el aire viciado llegue al salón.

Este primer análisis demuestra que Wright diseñó la vivienda siguiendo principios de arquitectura bioclimáticos, prediciendo una mejora sobre el confort térmico interior (7). Para poder comprobarlo de forma empírica se ha optado por la modelización y simulación energética (13) (14), empleando los programas Revit (15) para el modelo y Design Builder (16), que utiliza el motor de simulación de Energy Plus (17) (18) (19).

\section{METODOLOGÍA DE ANÁLISIS. MODELIZACIÓN PARAMÉTRICA Y DATOS DE PARTIDA}

El modelo realizado se sitúa en su ubicación original, Madison, Wisconsin, y el programa ha tomado el archivo de datos climáticos correspondientes a estas coordenadas para realizar las simulaciones.

La maqueta creada es fiel al diseño de Wright. Las características constructivas definidas en el análisis previo han sido introducidas en el programa Design Builder, aportando numerosos parámetros relevantes, como el valor de transmitancia térmica U de la envolvente. La vivienda, construida en 1937, no posee
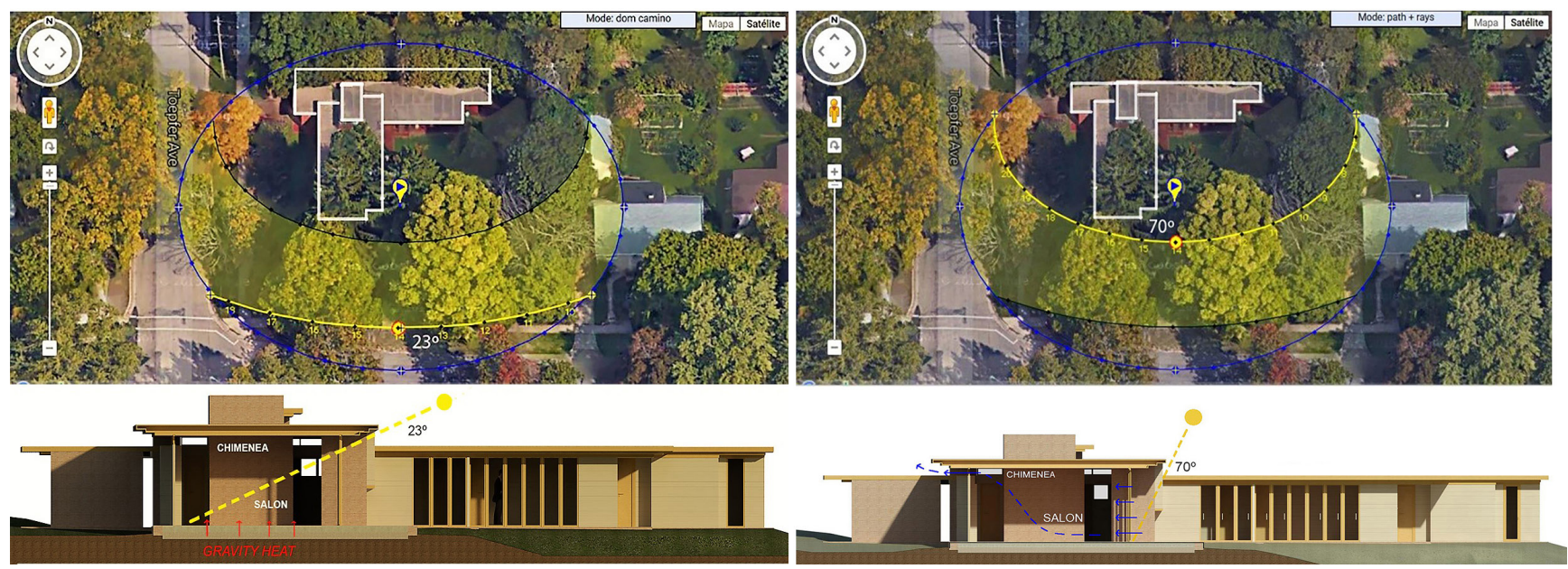

Figuras 6a y 6b. Estudio de soleamiento solsticio de invierno y de verano. 
aislamiento y los puentes térmicos, consecuencia del sistema constructivo empleado y del diseño del arquitecto son importantes, como se aprecia en los detalles constructivos (Figura 4).

La Tabla 1 muestra los valores aportados por el programa ( $\left.\mathrm{U}_{1}\right)$, los resultantes del cálculo de los coeficientes de la envolvente $\left(U_{2}\right)(20)$ y los valores máximos establecidos por el Código Técnico de la Edificación $\left(\mathrm{U}_{3}\right)$ (20), comprobando que los valores de la vivienda superan los máximos permitidos. Sin embargo, como se demuestra en el análisis que se presenta, a pesar de estas características, gracias a las estrategias bioclimáticas empleadas en el diseño de la vivienda, como la orientación y la ventilación natural, el diseño de los voladizos y el aprovechamiento de la inercia térmica de los materiales, Wright lograba una mejora considerable en cuanto a temperatura interior.

En cuanto a las instalaciones y confort térmico, como se ha expuesto en el análisis previo, en verano la vivienda original se sirve de la ventilación natural cruzada y nocturna para la disipación del calor interior, favorecida por la orientación y disposición de las ventanas. La separación de las fachadas opuestas es de 18'-o" (548 cm) tanto en el salón como en los dormitorios. La superficie de huecos de las fachadas este y sur, con ventanas de suelo a techo todas practicables, es de 72 y $48 \%$, respectivamente. Mientras que las fachadas oeste y norte con un 10 y $5 \%$ presentan una línea horizontal de ventanas practicables a una altura de 8'-o" (240 cm) y 6'-o" $(182 \mathrm{~cm})$. Para la simulación en verano se han establecido los siguientes criterios: las ventanas son abiertas por los usuarios cuando la temperatura interior de la vivienda supera los $24^{\circ}$ $\mathrm{C}$ y permanecen abiertas durante la noche en los meses de junio, julio y agosto en un $50 \%$ en el salón y dormitorios y un $100 \%$ en la cocina y baño. Durante el día, de 10:00 a 16:00, las ventanas permanecen cerradas evitando el sobrecalentamiento interior, y a partir de las 16:00 se abren al $100 \%$.
Para la simulación invernal se ha considerado la calefacción por suelo radiante definiendo una temperatura de consigna de $20^{\circ} \mathrm{C}$, dentro de los límites establecidos por el Reglamento de Instalaciones Térmicas de Edificios, RITE (21), y $2^{\circ} \mathrm{C}$ superior a lo establecido por el propio Wright en (11). Las simulaciones realizadas muestran que con esta consigna la temperatura interior máxima no llega a alcanzar $19^{\circ} \mathrm{C}$, por lo que se ha establecido que, durante la semana extrema de invierno con temperaturas exteriores entre $-1,22$ y $-23,87^{\circ}$ $\mathrm{C}$, la calefacción por suelo radiante permanece encendida las 24 horas del día. Para la simulación conjunta de la chimenea, ésta se ha considerado como un "proceso" eléctrico independiente, situado en el salón, con una densidad calórica de 300 $\mathrm{W} / \mathrm{m}^{2}$ y una potencia de $15 \mathrm{~kW}$ repartidos entre la superficie del salón, cuyo funcionamiento está relacionado con la programación de la ocupación de la vivienda. Cuando la chimenea se apaga, se detiene el proceso eléctrico. Para simular la inercia térmica de la masa de ladrillo de la chimenea el rendimiento del proceso se ha reducido de forma progresiva, 20 \%/hora, hasta llegar a o; chimenea apagada y ladrillos a temperatura ambiente. El efecto de la chimenea y de la inercia térmica de la masa de ladrillo sobre la temperatura interior se percibe en la Figura 7 de distribución de temperaturas y se traduce en las gráficas a través de bruscos cambios de temperatura, coincidiendo con el período en que está encendida.

\section{RESULTADOS Y DISCUSIONES}

Se han realizado simulaciones de la vivienda en diferentes escenarios teniendo en cuenta los resultados de temperatura operativa durante las semanas extremas del año; en invierno del 11-17 de enero, y en verano del 3-9 de julio. La comparación de estos resultados con los datos de la vivienda en su estado original va a permitir valorar y cuantificar la influencia de las estrategias pasivas de diseño bioclimático empleadas por Wright.

Tabla 1. Valores de transmitancia térmica $\mathrm{U}\left(\mathrm{W} / \mathrm{m}^{2} \mathrm{~K}\right) . \mathrm{U}_{1}$, datos de Design Builder.

$\mathrm{U}_{2}$, datos del cálculo analítico. $\mathrm{U}_{3}$, valores máximos según CTE (zona climática $\mathrm{D}$ ).

\begin{tabular}{|c|c|c|c|c|c|}
\hline & Materiales & Espesor & $\begin{array}{c}U_{1} \\
\left(W / \mathbf{m}^{2} K\right)\end{array}$ & $\begin{array}{c}U_{2} \\
\left(W / m^{2} K\right)\end{array}$ & $\begin{array}{c}\mathrm{U}_{3} \\
\left(\mathrm{~W} / \mathbf{m}^{2} \mathrm{~K}\right)\end{array}$ \\
\hline Fachada tipo 1 & 1 pie de ladrillo & 1'-o" $(30 \mathrm{~cm})$ & 1,68 & 1,91 & 0,60 \\
\hline Fachada tipo 2 & tablero-rastrel & $3 ”(7,5 \mathrm{~cm})$ & 1,38 & 1,64 & 0,60 \\
\hline Cubierta & Estructura de madera & 1'-0" $(30 \mathrm{~cm})$ & 0,90 & 1,68 & 0,40 \\
\hline Solera & Solera hormigón + grava & $4 "+4 ”(20 \mathrm{~cm})$ & 1,56 & 1,10 & 0,60 \\
\hline Ventanas & Acristalamiento sencillo & $4 \mathrm{~mm}$ & 5,77 & 5.7 & 2,70 \\
\hline
\end{tabular}

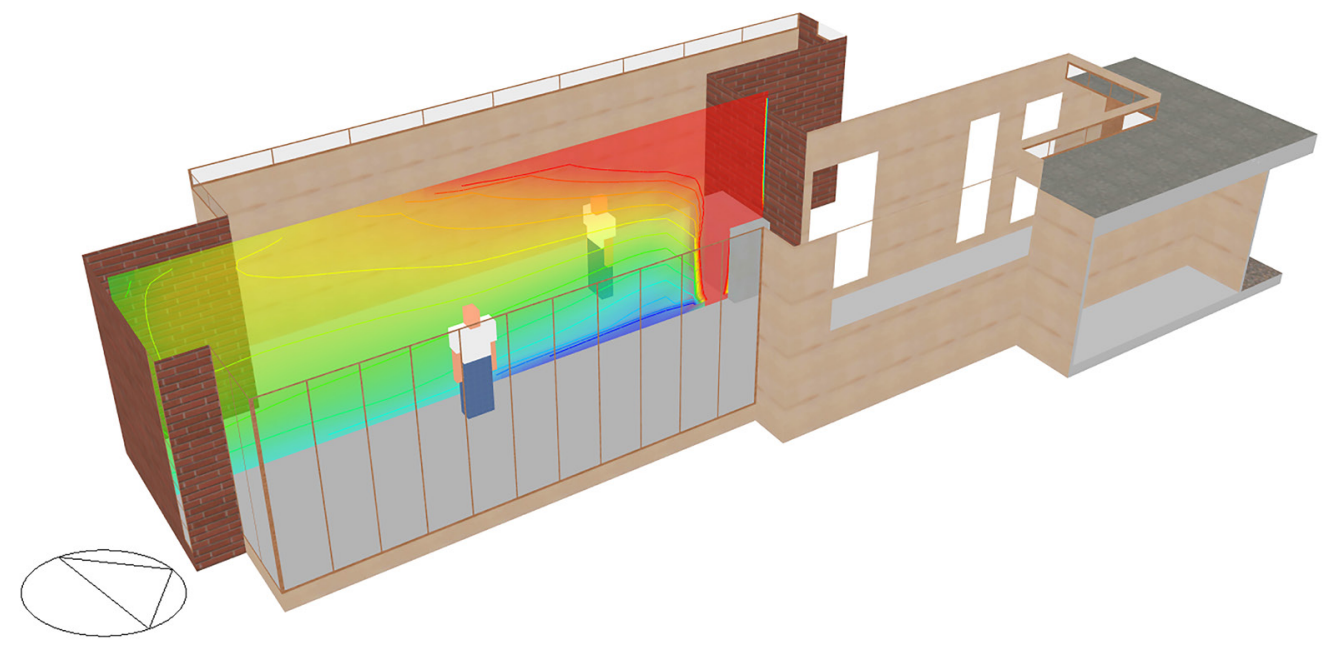

Figura 7. Distribución de temperaturas en el salón. Simulación chimenea y masa de ladrillo. 


\subsection{Influencia de la orientación}

El análisis realizado en el capítulo anterior muestra cómo la orientación original presenta teóricamente ventajas sobre el confort interior, tanto en verano como en invierno. Para comprobarlo se han llevado a cabo simulaciones con 8 orientaciones diferentes, partiendo de la original y girando la vivienda $45^{\circ}$ hasta alcanzar de nuevo la posición inicial.

\subsubsection{Verano}

La variable de la orientación influye sobre la temperatura interior en verano a través de dos factores: radiación solar y ventilación natural. Para cuantificar conjuntamente el efecto de la ventilación natural se han realizado las simulaciones de las orientaciones con y sin ventilación natural.

En este estudio se representan los resultados más característicos, comparando la orientación original con la $45^{\circ}$ y $270^{\circ}$.

La Gráfica 1a muestra los resultados de la influencia de la orientación sin tener la ventilación natural. Las temperaturas de la orientación original son más elevadas durante las primeras horas del día que el resto, casi todos los días, debido a la orientación este de sus superficies acristaladas. A medida que el sol avanza, la acción de los voladizos sobre la vivienda se

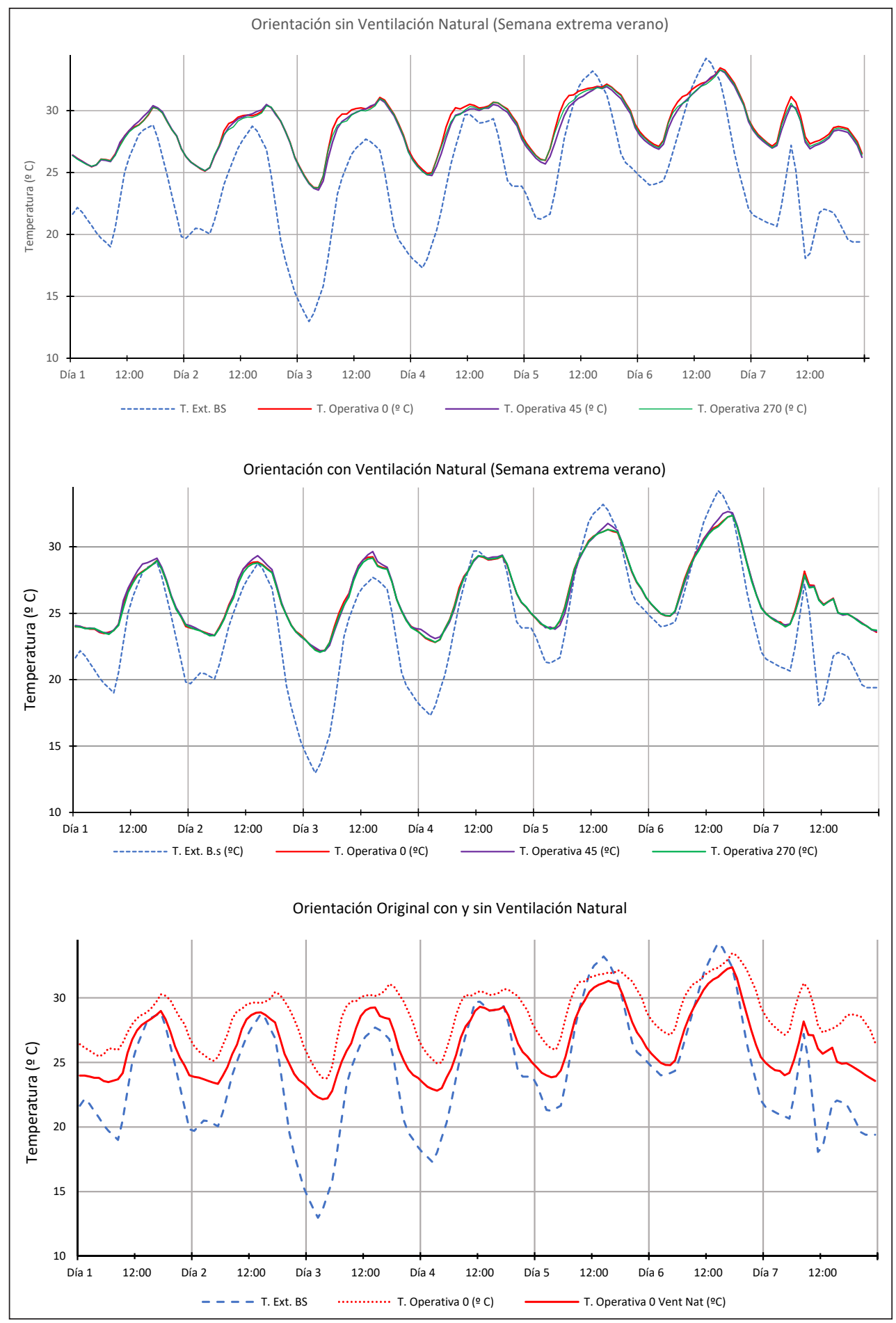

Gráfica 1a, 1b y 1c. Influencia orientación y ventilación natural sobre la temperatura interior. Verano. 
hace patente. Las temperaturas de las orientaciones $45^{\circ}$ y $270^{\circ}$ permanecen siempre ligeramente inferiores que las del estado original debido a los voladizos y a la orientación de las superficies acristaladas, respectivamente. Las temperaturas interiores máximas de todas las orientaciones alcanzan $30^{\circ} \mathrm{C}$ todos los días analizados, y presentan temperaturas superiores a $25^{\circ} \mathrm{C}$ durante toda la semana a excepción del día 3 de 4:00 a 5:00 h cuando las temperaturas exteriores descienden hasta $12,97^{\circ} \mathrm{C}$.

En la Gráfica 1b, teniendo en cuenta la ventilación natural, todas las orientaciones presentan datos de temperatura menores que la Gráfica 1a, superando los $30^{\circ} \mathrm{C}$ solamente los días 5 y 6 más calurosos. La temperatura operativa de la orientación original alcanza siempre los valores mínimos de temperatura máxima durante el día, considerándose la más favorable. La orientación $45^{\circ}$ presenta siempre temperaturas ligeramente superiores a la orientación original, con una diferencia de $0,60^{\circ} \mathrm{C}$ durante las horas más calurosas. Los resultados de la orientación $270^{\circ}$ son prácticamente idénticos a los de la original.

La acción de la ventilación natural es favorable en todas las orientaciones. La renovación del aire interior, junto con el efecto de la inercia térmica de la solera de hormigón y la masa de la fábrica de ladrillo de los muros y la chimenea, hace disminuir la temperatura en todos los casos. En el caso de la original, gracias al diseño y disposición de las ventanas en función de la dirección de los vientos dominantes, ésta juega un papel más efectivo, llegando a disminuir las temperaturas interiores hasta $4,55^{\circ} \mathrm{C}$ durante las últimas horas del día, como se aprecia en la Gráfica 1c.

Las Figuras 8a, 8b y 8c corresponden a la simulación de la ventilación natural en verano verificando el comportamiento del aire en el interior de la vivienda.

\subsubsection{Invierno}

Durante la semana de invierno se han realizado simulaciones teniendo en cuenta la calefacción por suelo radiante y la chimenea. La ventilación natural no se ha considerado dado su efecto negativo sobre la temperatura interior.

En la Gráfica 2 se aprecia que la orientación $45^{\circ}$ presenta temperaturas interiores ligeramente superiores a las de la orientación original los días 5 y 6 , con una diferencia máxima de casi $2^{\circ} \mathrm{C}$, día 6 a las 15:00 h, en que la temperatura exterior es de $-7,87^{\circ}$ C. Sin embargo, cuando las temperaturas exteriores descienden, incluso durante las horas del día, la diferencia entre las diferentes orientaciones es inapreciable, a excepción de la $270^{\circ}$ que presenta siempre los valores más desfavorables.

Analizando los resultados se observa que, aunque en invierno la orientación original presenta ocasionalmente temperaturas interiores ligeramente inferiores a la orientación $45^{\circ}$, en verano ésta resulta siempre la más favorable. Sin embargo, sería necesario analizar períodos más largos y realizar los análisis de soleamiento pertinentes para poder ratificar que la orientación elegida es la óptima durante todo el año.

\subsection{Influencia de los voladizos}

El primer análisis concluye que la dimensión y disposición de los voladizos favorece el confort interior en los meses calurosos de verano, sin detrimento en invierno. Para analizar este supuesto y medir su influencia sobre la temperatura interior se han realizado simulaciones con y sin voladizos, en verano y en invierno.

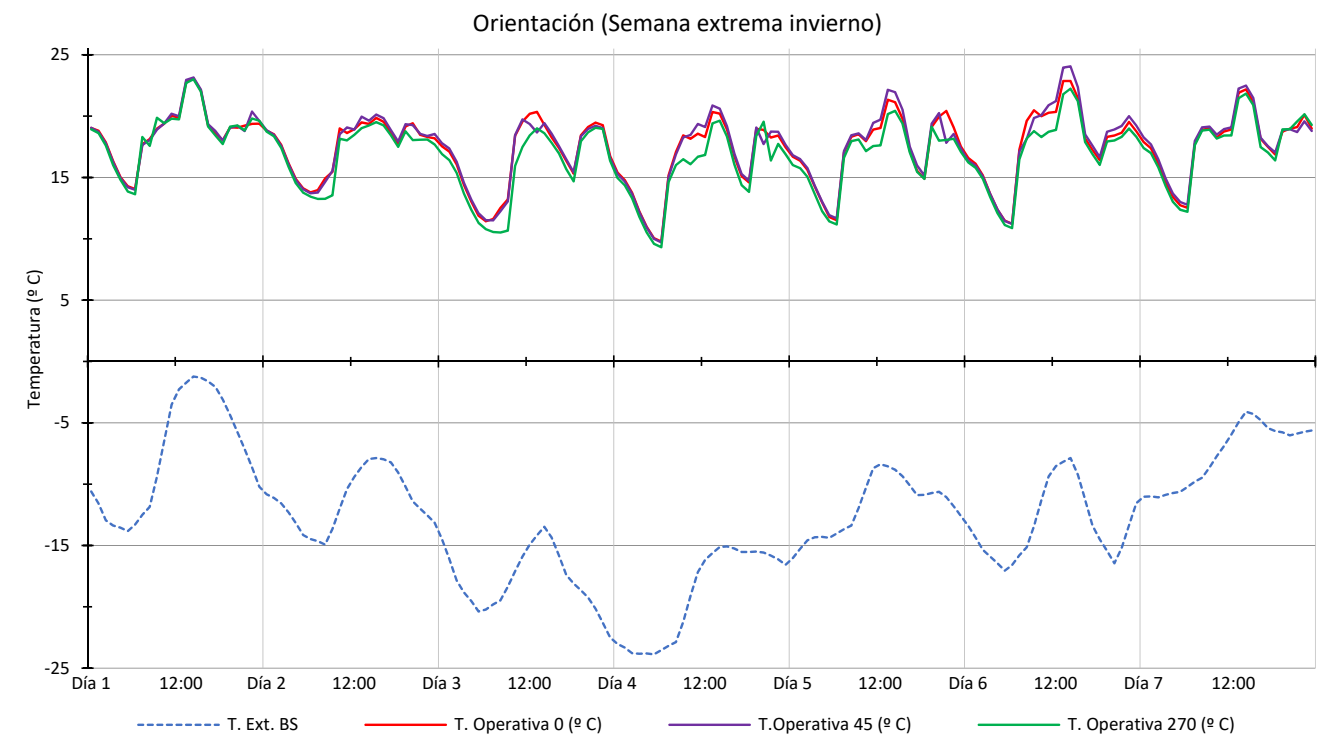

Gráfica 2. Influencia orientación sobre la temperatura interior. Invierno.
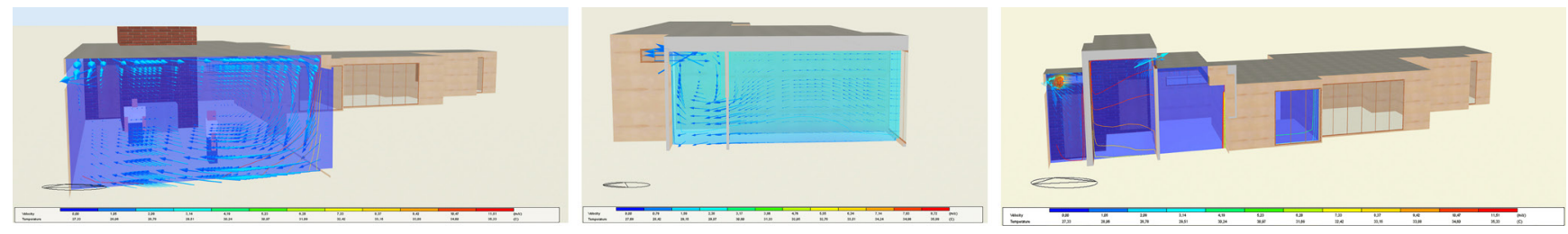

Figuras 8a, 8b y 8c. Ventilación natural en verano; salón, dormitorio y cocina. 
La Gráfica 3 muestra que la temperatura interior de la vivienda con voladizos presenta siempre datos más bajos, alcanzando una diferencia máxima de $2,50^{\circ} \mathrm{C}$ el día 3 a las 12:00 h.

Los resultados de las simulaciones de invierno con y sin voladizos son prácticamente iguales, demostrando que su situación y dimensión no presenta un efecto negativo sobre la temperatura interior, y permite que la vivienda se beneficie de la radiación solar durante el día.

\subsection{Influencia de la inercia térmica de los materiales empleados}

A priori, se ha supuesto que la inercia térmica de los materiales empleados por Wright, la fábrica de ladrillo de la chimenea y los muros de cerramiento, junto con el hormigón de la solera, disminuye el impacto de la fluctuación térmica exterior. Para medir su influencia sobre la temperatura interior y basándose en los estudios de Givoni (22), se han realizado tres simulaciones: estado original de la vivienda, un modelo con menor inercia térmica con todos los cerramientos exteriores y el forjado de planta baja de madera, y, por último, la opción de mayor inercia térmica sustituyendo los cerramientos originales de madera por fábrica de ladrillo (Tabla 2). Design Builder considera las propiedades térmicas de los materiales para las simulaciones, como conductividad, calor específico y emisividad (16).

En la Gráfica 4a se observa que la opción de vivienda con mayor inercia térmica presenta las menores temperaturas máximas y la menor oscilación día-noche. Mientras la vivienda de madera con menor inercia térmica presenta una mayor oscilación de temperaturas y las máximas más elevadas, con una diferencia con el estado original de hasta $2,80^{\circ} \mathrm{C}$ y con la opción de mayor inercia térmica de $4^{\circ} \mathrm{C}$. Las temperaturas del estado original son ligeramente más elevadas que las de la vivienda de ladrillo, alcanzando una diferencia máxima de hasta $1,20^{\circ} \mathrm{C}$, día 5 a las 15:00 h, siendo la temperatura exterior de $33,85^{\circ} \mathrm{C}$. En estas dos opciones el desfase de la onda térmica es de 4 horas en los días 5 y 6 más calurosos, sumado al efecto positivo de la ventilación natural consiguen reducir la temperatura máxima interior.

En la Gráfica 4b se observa que la opción de mayor inercia térmica presenta las temperaturas máximas menores con una diferencia máxima de 1,6 $6^{\circ} \mathrm{C}$ en relación con la vivienda original y de $4^{\circ} \mathrm{C}$ con la opción de menor inercia térmica. Sin embargo, presenta las mínimas más elevadas; de nuevo, menor oscilación. La opción con menor inercia térmica, gracias a la chimenea, alcanzando las temperaturas máximas más elevadas, con una diferencia máxima con la vivienda original de $2,5^{\circ} \mathrm{C}$, sin embargo, presenta las mínimas menores, siendo la oscilación térmica mayor. La gráfica de temperaturas del estado original, al igual que en verano, permanece entre las dos opciones. Hay que considerar que el consumo energético en calefacción es el mismo para las tres simulaciones.

Si bien en verano la vivienda de ladrillo podría considerarse la más favorable, en invierno presenta los datos más desfavorables. La opción con menor inercia térmica, favorecida por el efecto de la chimenea, presenta las temperaturas máximas más elevadas en invierno pero también en verano. La vivienda en su estado original, combinando de forma inteligente madera y ladrillo (Figura 9) presenta resultados medios, aprovechando las ventajas de los materiales tanto en verano como en invierno. La masa de ladrillo del núcleo central almacena el calor de la chimenea y lo cede al ambiente incluso cuando ésta se apaga. Los muros sur y la solera de hormigon, por su orientación, reciben radiación del sol en invierno

Tabla 2. Simulaciones realizadas para la influencia de la inercia térmica.

\begin{tabular}{|l|l|l|l|}
\hline Estado original (madera y ladrillo) & Madera & Ladrillo \\
\hline
\end{tabular}

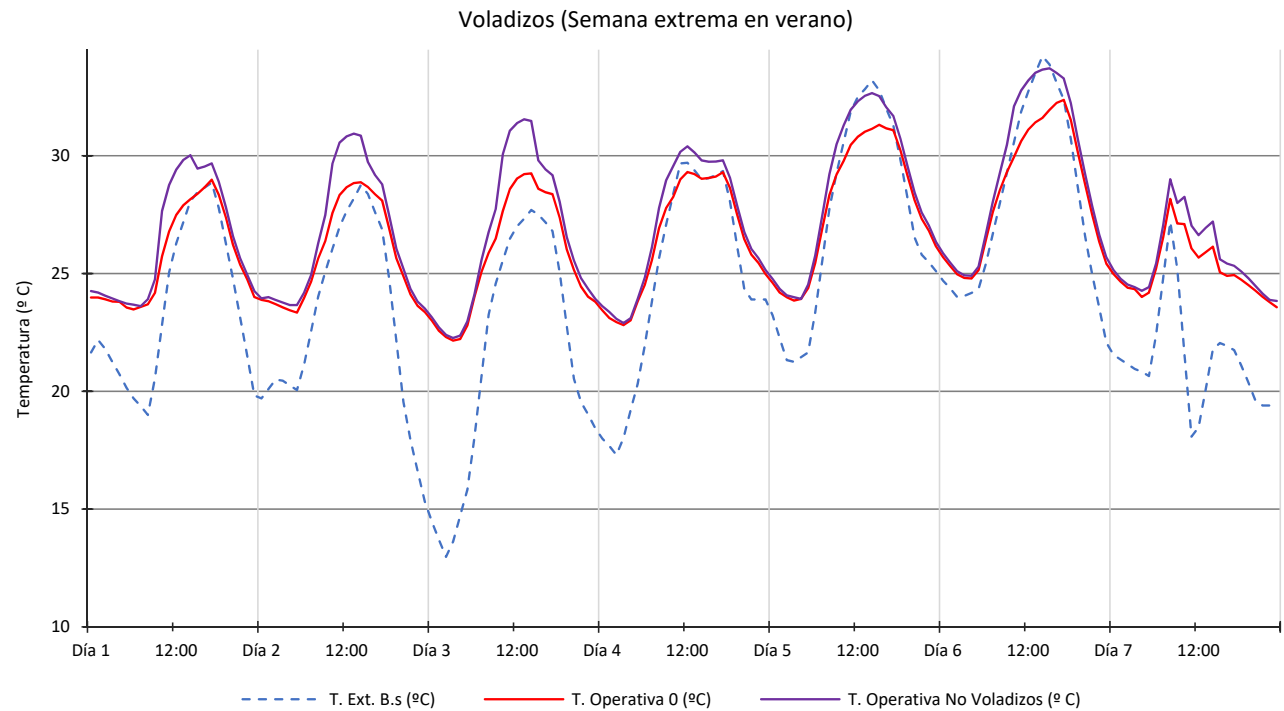

Gráfica 3. Influencia voladizos sobre la temperatura interior. Verano. 


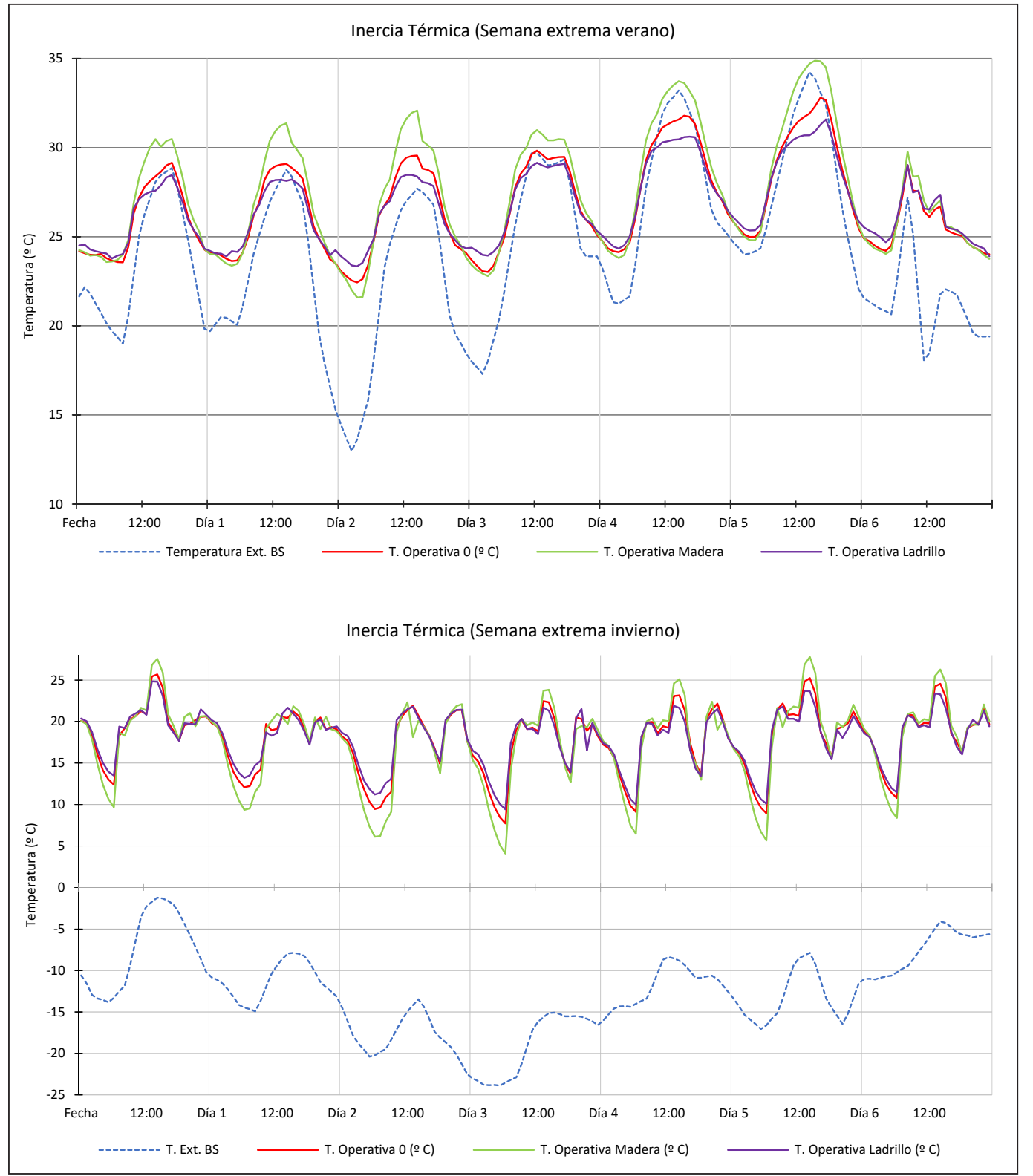

Gráfica 4a y 4b. Influencia inercia térmica de los materiales sobre la temperatura interior.

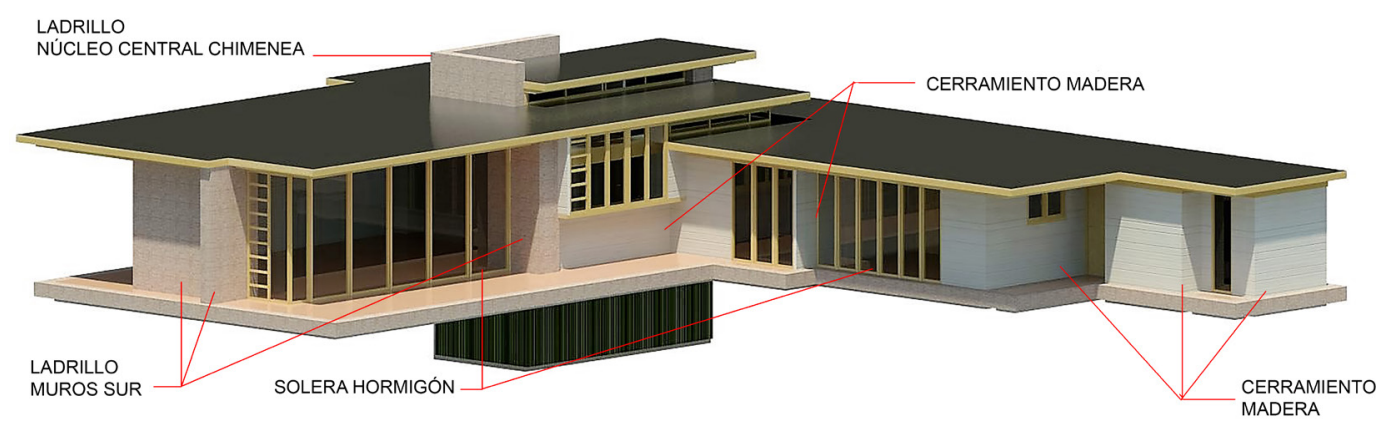

FACHADAS NORTE Y OESTE: CERRAMIENTO DE MADERA

Figura 9. Tipos de cerramiento.

prácticamente todo el día, emitiéndolo al interior durante el atardecer. En verano el núcleo central en el interior de la vivienda no recibe radiación solar, y los voladizos protegen los muros sur y la solera. Durante la noche estos elementos, que no se han sobrecalentado, se enfrían gracias a la ventilación nocturna, refrescando la vivienda durante la mañana.
A continuación se presenta la Tabla 3 con los resultados más representativos de cada simulación. Se ha señalado la diferencia de temperatura interior de las variables analizadas, destacando la acción de la ventilación natural sobre la temperatura interior que supone un descenso de hasta $4,5^{\circ}$ en los días calurosos de verano. 
Tabla 3. Resumen de resultados más representativos.

\begin{tabular}{|c|c|c|c|c|c|c|c|c|}
\hline \multirow[t]{3}{*}{ Variables } & \multirow[t]{3}{*}{ Fecha } & \multirow{2}{*}{\begin{tabular}{|c|}
$\begin{array}{c}\text { Estado } \\
\text { original }\end{array}$ \\
T. interior \\
\end{tabular}} & \multicolumn{3}{|c|}{ Simulación 2} & \multicolumn{3}{|c|}{ Simulación 3} \\
\hline & & & Descripción & T. interior & Diferencia & Descripción & T. interior & Diferencia \\
\hline & & $\mathrm{T}(\mathbf{O} \mathbf{C})$ & & $\mathbf{T}\left(\mathbf{O}^{\mathrm{C}}\right)$ & $\Delta \mathrm{T}\left({ }^{\circ} \mathrm{C}\right)$ & & $\mathbf{T}\left({ }^{\circ} \mathbf{C}\right)$ & $\Delta \mathrm{T}\left({ }^{\circ} \mathrm{C}\right)$ \\
\hline $\begin{array}{l}\text { Ventilación } \\
\text { natural }\end{array}$ & Verano Día 3-21:0o h & 25,16 & $\begin{array}{c}\text { Sin } \\
\text { ventilación } \\
\text { natural }\end{array}$ & 29,71 & 4,55 & & & \\
\hline Voladizos & Verano Día 3-21:oo h & 25,58 & Sin voladizos & 31,05 & 2,47 & & & \\
\hline $\begin{array}{l}\text { Inercia } \\
\text { térmica }\end{array}$ & Verano Día 6-15:0o h & 31,90 & De madera & 34,70 & 2,80 & De ladrillo & 30,65 & $-1,25$ \\
\hline $\begin{array}{l}\text { Inercia } \\
\text { térmica }\end{array}$ & Invierno Día 5-15:0o h & 25,23 & De madera & 27,80 & 2,57 & De ladrillo & 23,65 & $-1,58$ \\
\hline
\end{tabular}

\section{CONCLUSIONES}

Las siguientes conclusiones deben entenderse en su contexto histórico. Las estrategias bioclimáticas empleadas por Wright en la casa Jacobs, en muchos casos herederas de antiguas tradiciones arquitectónicas, supusieron mejoras en el comportamiento térmico del edificio. Consideradas como novedosas en la construcción de vivienda norteamericana de los años 30, muchas de ellas han sido plenamente asumidas por la arquitectura bioclimática actual. El presente estudio analiza, mediante herramientas de simulación modernas, la influencia de las estrategias bioclimáticas sobre el confort interior tomando la temperatura operativa como referencia.

1. La modelización paramétrica permite cuantificar la acción de las estrategias pasivas empleadas en la vivienda. Las principales variables estudiadas y sus ventajas son las siguientes:

- Las grandes superficies acristaladas representan un papel fundamental en la calefacción natural en invierno gracias a la orientación de la vivienda, sur y este. Sin embargo, en verano están protegidas por los grandes voladizos evitando el sobrecalentamiento interior, suponiendo una reducción de la temperatura interior de $2,5^{\circ} \mathrm{C}$.

- Las ventanas, al ser practicables y gracias a la orientación adecuada, permiten la ventilación refrescando el interior cuando las temperaturas exteriores descienden. La reducción de la temperatura interior en verano generada mediante esta estrategia puede estimarse en casi $5^{\circ} \mathrm{C}$.
- Wright utilizó cerramientos de diferentes inercias (ladrillo o madera) en función de las orientaciones. El análisis realizado muestra el beneficio de esta estrategia en el comportamiento térmico del edificio mediante el balance de sus efectos a lo largo de las diferentes estaciones.

2. La vivienda construida en 1937 empleaba materiales con valores de transmitacia térmica U muy por debajo de los exigidos en la actualidad. A pesar de ello, como demuestran los resultados obtenidos, gracias a las estrategias bioclimáticas como la orientación y la ventilación natural, el diseño de voladizos y el aprovechamiento de la inercia térmica de los materiales, Wright consigue una mejora sobre la temperatura interior tanto en verano como en invierno. La aplicación de estos conceptos sobre la construcción actual de viviendas, que emplea sistemas constructivos más evolucionados, supondría un resultado muy efectivo al apoyarse sobre una base mucho mejor.

3. Del mismo modo, el empleo de estas estrategias pasivas puede, en determinados contextos, reducir el uso de sistemas de climatización mecánicos con el correspondiente ahorro energético que esto supondría.

4. El análisis realizado muestra que la calidad espacial y formal de la obra de Frank Lloyd Wright es, en numerosas ocasiones, el resultado de una reflexión global junto a la preocupación por el confort interior teniendo en cuenta el entorno y la naturaleza.

\section{REFERENCIAS}

(1) Banham, R. (1969). The architecture of the well-tempered environment, p. 70. London: The Architectural Press, doi: http://dx.doi.org/10.1016/B978-0-85139-074-1.50009-1.

(2) Jiménez, I. (2011). Frank Lloyd Wright: A Precursor of Sustainable Architecture. Americas, 63(5): 6. Organisation of the American States.

(3) Wright, F. L. (1943). An Autobiography, p. 349. San Francisco: Pomegranate Communications, Inc.

(4) García, J. (2010). ¿Sostenibilidad?... o Simplemente Sensatez. Informes de la Construcción, 62(517): 4, doi: https://doi. org/10.3989/ic.2010.v62.i517.

(5) Jacobs, H. (1978). Building with Frank Lloyd Wright: An Illustrated Memoir, p. 5o. San Francisco: Chronicle Books.

(6) Datos climáticos de Madison, Wisconsin. https://www.windfinder.com/forecast/university-of-wisconsin-madison. Consulta realizada el 15-04-2015.

(7) Beltrán, M. A., García, J., Dufrasnes, E. (2015, 17 octubre). La casa Jacobs I de Frank Lloyd Wight. Un hito en la arquitectura bioclimática. En Noveno Congreso Nacional y Primero Internacional Hispanoamericano de Historia de la Construcción (pp. 199-209). Segovia: Instituto Juan de Herrera.

(8) United States Department of the Interior, National Historic Landmark Nomination. (2003). National Register of Historic Places Registration Form: Jacobs, Herbert and Katherine, First House. Washington DC: National Park Service.

(9) Del Río, M., Salto Weis, I., Izquierdo, P. (2010). Sustainable Construction: Construction and Demolition Waste Reconsidered. Waste Management \& Research, 28(2): 118-129, doi: http://dx.doi.org/10.1177/0734242Xo9103841. 
(10) Siry, J. M. (2013). Frank Lloyd Wright's innovative approach to environmental control in his buildings for the S. C. Johnson Company. Construction History, 28(1): 141-164.

(11) Wright, F. L. (1954). The Natural House, p. 99-10o. Nueva York: Horizon Press.

(12) Sturgeon, A., Porges, M. (1997-1998). The Vital Signs Case Study- Building with Frank Lloyd Wright. Milwaukee: Universidad de Wisconsin.

(13) Schiler, M., Brahmbhatt. S. (2006, 6-8 septiembre). Thermal Comfort in a Sustainable House by Frank Lloyd Wright. En The $23^{\text {rd }}$ Conference on Passive and Low Energy Architecture. Ginebra: Universidad de Ginebra.

(14) Douglass, C. D. (2010). Instructional Modules Demonstrating Building Energy Analysis Using a Building Information Model (Tesis). Urbana: Universidad de Illinois.

(15) Autodesk, Revit. http://sustainability.autodesk.com/available-solutions/rapid-energy-modeling/.

(16) Design Builder. https://www.designbuilder.co.uk/.

(17) Sabol, L. (2008 noviembre). Measuring Sustainability for Existing Buildings. IFMA World Workplace. Dallas: Design + Construction Strategies.

(18) Geva, A. (2012). Frank Lloyd Wright's Sacred Architecture. Faith, Form and Building Tecchnology, p. 244,245. Londres y Nueva York: Routledge.

(19) Crawley, D., et al. (2008). Contrasting the capabilities of building energy performance simulation programs. Building and Environment, 43: 661-673, doi: http://dx.doi.org/10.1016/j.buildenv.2006.10.027.

(20) Ministerio de Fomento (2016). Código Técnico de la Edificación Documento Básico Ahorro de Energía.

(21) Boletín Oficial del Estado (2007). Real Decreto 1027/2007, Reglamento de Instalaciones Térmicas en los Edificios (RITE). BOE, $\mathrm{n}^{\circ}$ 207. España.

(22) Givoni, B. (2011). Indoor temperature reduction by passive cooling systems. Solar Energy, 85: 1692-1726, doi: http:// dx.doi.org/10.1016/j.solener.2009.10.003. 